Original Article

\title{
Electromyographic analysis of the infraspinatus and scapular stabilizing muscles during isometric shoulder external rotation at various shoulder elevation angles
}

\author{
Daisuke UGa, RPT, MS ${ }^{1,2)^{*}}$, Yasuhiro Endo, RPT, PhD ${ }^{1)}$, Rie Nakazawa, RPT, PhD ${ }^{2)}$, \\ MasaAki SAKAmoto, RPT, $\mathrm{PhD}^{2)}$ \\ 1) Jobu Hospital for Respiratory Diseases: 586-1 Taguchi, Maebashi, Gunma 371-0048, Japan \\ 2) Graduate School of Health Sciences, Gunma University, Japan
}

\begin{abstract}
Purpose] This study aimed to clarify activation of the infraspinatus and scapular stabilizing muscles during shoulder external rotation at various shoulder elevation angles. [Subjects] Twenty subjects participated in this study and all measurements were performed on the right shoulder. [Methods] Isometric shoulder external rotation strength and surface electromyographic data were measured with the shoulder at $0^{\circ}, 45^{\circ}, 90^{\circ}$, and $135^{\circ}$ elevation in the scapular plane. The electromyographic data were collected from the infraspinatus, upper trapezius, middle trapezius, lower trapezius, and serratus anterior muscles. These measurements were compared across the various shoulder elevation angles. [Results] The strength measurements did not differ significantly by angulation. The infraspinatus activity was $92 \%, 75 \%, 68 \%$, and $57 \%$ of the maximum voluntary contraction, which significantly decreased as shoulder elevation increased. The serratus anterior activity was $24 \%, 48 \%, 53 \%$, and $62 \%$ of the maximum voluntary contraction, which significantly increased as shoulder elevation increased. [Conclusion] Shoulder external rotation torque was maintained regardless of shoulder elevation angle. The shoulder approximated to the zero position as the shoulder elevation increased so that infraspinatus activity decreased and the scapular posterior tilting by the serratus anterior might generate shoulder external rotation torque.

Key words: Shoulder external rotation, Infraspinatus, Serratus anterior
\end{abstract}

(This article was submitted Sep. 9, 2015, and was accepted Oct. 14, 2015)

\section{INTRODUCTION}

For overhead sports athletes, shoulder injuries are severe problems that influence the length of the player's sports career and can affect the activities of daily living. The rotator cuff plays a vital role during shoulder motion by maintaining stability of the humeral head in the scapular glenoid ${ }^{1)}$. The shoulder external rotator muscles (e.g., the infraspinatus [ISP] and teres minor muscles) are breaking muscles for shoulder traction force, horizontal adduction, and internal rotation during the deceleration phase of the throwing motion and maintain the centripetal position of the humeral head. Dysfunction of these muscles involves internal impingement, shoulder instability, labral lesions, and rotator cuff lesions ${ }^{2}$. For overhead sports athletes, shoulder external rotator muscle weakness is often recognized to be a problem ${ }^{3,4)}$, and many authors have emphasized the need to enhance shoulder external rotation muscular strength and endurance during rehabilitation and conditioning programs $^{3-5}$ ). The shoulder joint is one of the most mobile joints in the human body, and muscular function seems to vary among shoulder positions. However, it remains unclear how muscular function changes in response to shoulder elevation angle changes during shoulder external rotation.

\footnotetext{
*Corresponding author. Daisuke Uga (E-mail: m11711024@gunma-u.ac.jp)

(C)2016 The Society of Physical Therapy Science. Published by IPEC Inc.

This is an open-access article distributed under the terms of the Creative Commons Attribution Non-Commercial No Derivatives (by-nc-nd) License $<$ http://creativecommons.org/licenses/by-nc-nd/3.0/>.
} 
The scapula plays various roles in achieving appropriate motion and positions to facilitate efficient physiology and biomechanics to optimize shoulder function ${ }^{6}$. The scapula acts as a base for muscle attachment and moves in a coordinated manner with the moving humerus so that the instant center of rotation is constrained within a physiological pattern through the full range of shoulder motion. Scapular dysfunction or abnormal motion (e.g., scapular instability, scapular dyskinesis) has recently attracted attention and is believed to be involved in subacromial impingement, rotator cuff disease, glenohumeral joint instability, and other shoulder problems ${ }^{7,8)}$. Scapular function affects rotator cuff function ${ }^{9-11)}$, because the rotator cuff attaches to the scapula. Scapular dysfunction involves tightness of the pectoralis minor and posterior glenohumeral joint soft tissue, thoracic kyphosis or a flexed posture, and weakness and abnormal activity of the scapular stabilizing muscles ${ }^{8)}$. Researchers believe that inadequate serratus anterior (SA), lower trapezius (LT) activation and excess upper trapezius (UT) activation are problems during shoulder elevation in pathological states ${ }^{11)}$. However, these reports of scapular stabilizing muscle activation have focused on shoulder elevation motion, and muscular activation of the scapular stabilizing muscles during shoulder external rotation requires clarification.

Therefore, the purpose of this study was to clarify ISP and scapular stabilizing muscle activation during shoulder external rotation at various shoulder elevation angles by using surface electromyography (EMG).

\section{SUBJECTS AND METHODS}

Twenty subjects participated in this study; the right shoulder of each subject was studied. The subjects were healthy men with a mean age of $22.4 \pm 1.5$ years, height of $172.9 \pm 4.4 \mathrm{~cm}$, and weight of $67.1 \pm 5.8 \mathrm{~kg}$. The target muscles were the ISP, UT, middle trapezius (MT), LT, and SA. The subjects were given descriptions of the purpose, content, and methods of this study, and each provided written consent for participation. This study met the institutional ethical requirements for human experimentation of the Declaration of Helsinki.

For collecting the EMG data, data acquisition hardware (PowerLab 16/35; AD Instruments Japan, Aichi, Japan) was used, with a sampling rate of 1,000 Hz. Surface electrodes (DL-141 active electrode; S\&ME Inc., Tokyo, Japan) were used and attached to each muscle after appropriate skin preparation. The EMG electrodes positions were as described in a previous study $^{12-14)}$. For the ISP, the electrode was placed two fingerbreadths inferior to the center of the spina scapulae. For the UT, the electrode was placed midway between the spinous process of the seventh cervical vertebra and the posterior tip of the acromion process along the line of the trapezius. For the MT, the electrode was placed midway on a horizontal line between the root of the spine of the scapula and the third thoracic spine. For the LT, the electrode was placed midway between the spinous process of the seventh thoracic vertebrae and the vertebral border of the scapula at the junction of the scapular spine. For the SA, the electrode was placed on the midaxillary line at the level of the inferior angle of the scapula.

The subjects engaged in an adequate warm-up of the neck and shoulder, and then EMG data during maximum voluntary contraction (MVC) of each muscle were collected. The isometric shoulder external rotation task was taken in four shoulder elevation angles (i.e., shoulder $0^{\circ}, 45^{\circ}, 90^{\circ}$, and $135^{\circ}$ elevation in the scapular plane) with a neutral position of shoulder rotation, fore arm pronation/supination, and elbow flexed at $90^{\circ}$. At each angle, the muscular strength measurement with a hand- held dynamometer ( $\mu$-Tas MF-01; ANIMA Corp., Tokyo, Japan) and EMG data recording were performed simultaneously for $5 \mathrm{~s}$ and repeated three times. Subjects were seated in a chair, and the trunk was fixed to the chair with a belt to minimize the effect of each individual's trunk function. Moreover, the distal portion of the upper arm was set on a stable table to minimize the effect of muscle activity for maintaining the shoulder position. When subjects performed compensatory movements, the measurements were stopped and restarted after a rest period.

The obtained raw EMG data were processed using biological waveform analysis software (LabChart7; AD Instruments Japan, Aichi, Japan). Raw EMG data were filtered with a digital band-pass filter between 20 and $500 \mathrm{~Hz}$, and root mean square values were obtained for $3 \mathrm{~s}$ in the middle, excluding $1 \mathrm{~s}$ each at the beginning and at the end of the EMG recording. The $\%$ MVC value was calculated by normalizing the muscle activity of each muscle in isometric shoulder external rotation with the MVC.

The statistical analyses were performed with SPSS version 21.0 for Windows (SPSS, Chicago, IL, USA). Shoulder external rotation strength and muscle activity were compared at each shoulder elevation angle using repeated-measures analysis of variance. If a significant shoulder elevation angle effect was present, the differences were evaluated using a t-test with a Bonferroni-correction, and significance was set at $5 \%$.

\section{RESULTS}

Isometric shoulder external rotation strength and the normalized EMG activity of each muscle are shown in Tables 1 and 2. The shoulder external rotation strength did not differ significantly among the shoulder elevation angles. The ISP activity was significantly higher at the $0^{\circ}$ than at all of the other angles $(\mathrm{p}<0.01)$, higher at $45^{\circ}$ than at $135^{\circ}(\mathrm{p}<0.01)$, and higher at $90^{\circ}$ than at $135^{\circ}(\mathrm{p}<0.05)$. The UT activity showed no significant differences among the shoulder elevation angles. The MT activity was significantly higher at $45^{\circ}$ than at $135^{\circ}(\mathrm{p}<0.05)$. The LT activity was significantly higher at $0^{\circ}$ than at $90^{\circ}(\mathrm{p}<0.05)$ and at $135^{\circ}(\mathrm{p}<0.01)$, and higher at $45^{\circ}$ than at $135^{\circ}(\mathrm{p}<0.05)$. The SA activity was significantly lower at $0^{\circ}$ than at all of the other angles $(\mathrm{p}<0.01)$, and lower at $45^{\circ}$ than at $135^{\circ}(\mathrm{p}<0.05)$. 
Table 1. Isometric shoulder external rotation strength at various shoulder elevation angles

\begin{tabular}{lcccc}
\hline & \multicolumn{4}{c}{ Shoulder elevation angles in the scapular plane } \\
\cline { 2 - 5 } & $0^{\circ}$ & $45^{\circ}$ & $90^{\circ}$ & $135^{\circ}$ \\
\hline Shoulder ER strength $(\mathrm{N})$ & $94.1(12.0)$ & $100.5(21.0)$ & $97.3(19.5)$ & $93.5(20.1)$ \\
\hline
\end{tabular}

Data are shown as means (standard deviation). No significant difference is noted among the various shoulder elevation angles. ER: external rotation

Table 2. Muscle activities (\% maximum voluntary contraction) during isometric shoulder external rotation at various shoulder elevation angles

\begin{tabular}{|c|c|c|c|c|}
\hline & \multicolumn{4}{|c|}{ Shoulder elevation angles in the scapular plane } \\
\hline & $0^{\circ}$ & $45^{\circ}$ & $90^{\circ}$ & $135^{\circ}$ \\
\hline Infraspinatus & $91.6(20.3)$ & $74.6(18.9)^{* *}$ & $68.0(25.0)^{* *}$ & $57.1(16.8)^{* *+\dagger \S}$ \\
\hline Upper trapezius & $29.0(21.6)$ & $30.0(11.5)$ & $35.6(20.7)$ & $38.1(20.4)$ \\
\hline Middle trapezius & $56.1(26.9)$ & $60.0(29.4)$ & $46.8(19.9)$ & $42.0(18.3)^{\dagger}$ \\
\hline Lower trapezius & $74.0(29.5)$ & $70.5(27.6)$ & $62.5(21.8)^{*}$ & $54.7(18.6)^{* * \dagger}$ \\
\hline Serratus anterior & $23.6(15.6)$ & $48.1(15.5)^{* *}$ & $52.7(21.3)^{* *}$ & $62.4(22.5)^{* * \dagger}$ \\
\hline
\end{tabular}

compared with " $45^{\circ}$ "; ${ }^{\S} \mathrm{p}<0.05$, compared with " $90^{\circ}$ ",

\section{DISCUSSION}

The rotator cuff plays a critical role in stabilizing the glenohumeral joint ${ }^{1)}$ for normal shoulder function. In overhead sports athletes like baseball pitchers, weakness of the shoulder external rotator is often related to shoulder injury ${ }^{3,4)}$, and shoulder external rotation strengthening is one of the most important programs during rehabilitation or athletic conditioning ${ }^{3-5}$. The shoulder joint is one of the most mobile joints in the human body, and its related muscular function seems to change in various shoulder positions. In this study, isometric shoulder external rotation strength at four shoulder elevation angles (i.e., shoulder $0^{\circ}, 45^{\circ}, 90^{\circ}$, and $135^{\circ}$ elevation in the scapular plane) showed no significant differences among the various angles. This finding suggests that shoulder external rotation torque in various shoulder elevations in the scapular plane is constantly maintained. Moreover, after consideration of muscle positional relationships and change in muscular direction, we concluded that the source of the shoulder external rotation torque generation differ at each shoulder elevation angle.

The ISP is more active than the other rotator cuff muscles and the deltoid in shoulder external rotation ${ }^{15)}$. In this study, the ISP was a prime mover of shoulder external rotation, but muscle activity decreased when shoulder elevation angle increased. Kuechle ${ }^{16)}$ reported that the ISP is the most efficient external rotator, regardless of position, followed by the teres minor, posterior deltoid (neutral position), and supraspinatus (coronal position) by the potential moment (the product of moment arm, physiologic cross-sectional area, and a constant relating cross-sectional area to muscle force; i.e., potential moment $=$ physiological cross-sectional area $\times$ constant $\left[4.7 \mathrm{~kg} / \mathrm{cm}^{2}\right] \times$ moment arm) . Moreover, because of the physiological crosssectional area of the ISP, which is larger than the teres minor, the potential moment of the ISP is larger than that of the teres minor at $0^{\circ}$ and $90^{\circ}$ shoulder elevation in the scapular plane. In this study, although shoulder external rotation torque was maintained regardless of shoulder elevation angle, the ISP activity decreased when the shoulder elevation angle was increased. Although involvement of the teres minor, which was not measured in this study, was contemplated, considering the potential moment (ISP $>$ teres minor), this effect could not be explained. Therefore, the effect was considered to be due to the influence of muscles other than the ISP and teres minor.

Trapezius activity increases with increasing shoulder elevation angle ${ }^{17}$. In this study, the distal portion of the upper arm was set on a stable table to minimize the effect of muscle activity required to maintain the shoulder elevation. Therefore, the UT activity showed no significant differences among the shoulder elevation angles. Similarly, the MT and LT activities decreased rather than increased as the shoulder elevation angle increased. Since these results are similar to the change in ISP activity, these changes suggest that the MT and LT were active as a force against the outside excursion of the scapula due to the ISP in shoulder external rotation.

SA activity increased as the shoulder elevation angle increased, showing the most characteristic change in the scapular stabilizing muscle. As previously mentioned, in this study, because muscle activity required to maintain shoulder elevation was minimized, this change was regarded as the increase involved in shoulder external rotation. With the shoulder elevated at $135^{\circ}$, it approximates to the position called zero position. In the zero position, because the directions of the rotator cuff and humerus axis coincide, humerus rotation is less likely to occur ${ }^{18)}$. As the shoulder external rotation strength showed no significant differences among the shoulder elevation angles, shoulder external rotation torque in the shoulder at $135^{\circ}$ 
elevation was considered maintained. The SA creates scapular posterior tilt ${ }^{19}$ ). Therefore, in shoulder elevation positioning, scapular posterior tilting by the SA was considered to generate the shoulder external rotation torque.

The above findings suggested that, although the ISP acted as a prime shoulder external rotator, scapular posterior tilting by the SA also generated shoulder external rotation torque as the shoulder elevation angle increased. In the clinical setting, it is important to consider evaluation and training of the shoulder function to account for this difference. In particular, overhead sports athletes who often use their arms in elevated positions should have their SA function thoroughly evaluated. Although this study targeted the healthy men, it is necessary to consider its relevance to shoulder injuries in overhead sports athletes. Moreover, it is necessary to accumulate evidence to prevent shoulder injury and create rehabilitation programs to enable them to return to competitions.

A limitation of this study was that an isometric task was adopted in this study, although the shoulder external rotator is often needed as an eccentric contraction in sports activities. Therefore, it is impossible to measure the effects of differences in contraction types and motion velocity. Further, since a kinematic analysis was not performed, shoulder external rotation torque of scapular posterior tilting by the SA was not studied. In this study, only the superficial muscles were targeted; as such, the effect of deep muscles such as the supraspinatus, teres minor, rhomboid, and levator scapulae were not evaluated. Intramuscular electrodes are necessary for these deep muscle analyses. Therefore, in the future, it will be important to reconsider the SA function for shoulder external rotation in shoulder elevation positions by considering electrode types and movement tasks.

\section{REFERENCES}

1) Phadke V, Camargo P, Ludewig P: Scapular and rotator cuff muscle activity during arm elevation: a review of normal function and alterations with shoulder impingement. Rev Bras Fisioter, 2009, 13: 1-9. [Medline] [CrossRef]

2) Werner SL, Gill TJ, Murray TA, et al.: Relationships between throwing mechanics and shoulder distraction in professional baseball pitchers. Am J Sports Med, 2001, 29: 354-358. [Medline]

3) Wilk KE, Andrews JR, Arrigo CA, et al.: The strength characteristics of internal and external rotator muscles in professional baseball pitchers. Am J Sports Med, 1993, 21: 61-66. [Medline] [CrossRef]

4) Wilk KE, Meister K, Andrews JR: Current concepts in the rehabilitation of the overhead throwing athlete. Am J Sports Med, 2002, 30: 136-151. [Medline]

5) Lin HT, Ko HT, Lee KC, et al.: The changes in shoulder rotation strength ratio for various shoulder positions and speeds in the scapular plane between baseball players and non-players. J Phys Ther Sci, 2015, 27: 1559-1563. [Medline] [CrossRef]

6) Kibler WB: The role of the scapula in athletic shoulder function. Am J Sports Med, 1998, 26: 325-337. [Medline]

7) Ludewig PM, Cook TM: Alterations in shoulder kinematics and associated muscle activity in people with symptoms of shoulder impingement. Phys Ther, 2000, 80: 276-291. [Medline]

8) Ludewig PM, Reynolds JF: The association of scapular kinematics and glenohumeral joint pathologies. J Orthop Sports Phys Ther, 2009, 39: 90-104. [Medline] [CrossRef]

9) Merolla G, De Santis E, Sperling JW, et al.: Infraspinatus strength assessment before and after scapular muscles rehabilitation in professional volleyball players with scapular dyskinesis. J Shoulder Elbow Surg, 2010, 19: $1256-1264$. [Medline] [CrossRef]

10) Jung J, Cho K, Yu J: Effects of scapular stabilizing exercise in patients with partial-thickness rotator cuff tear. J Phys Ther Sci, 2012, 24: 1173-1175. [CrossRef]

11) Joshi M, Thigpen CA, Bunn K, et al.: Shoulder external rotation fatigue and scapular muscle activation and kinematics in overhead athletes. J Athl Train, 2011, 46: 349-357. [Medline]

12) Kai $Y$, Gotoh $M$, Nagata $K$, et al.: Infraspinatus fatigue during resisted arm elevation with isometric contraction: an electromyographic study. J Shoulder Elbow Surg, 2012, 21: 1104-1109. [Medline] [CrossRef]

13) Choi Y, Chung SH, Shim JH: Comparisons of shoulder stabilization muscle activities according to postural changes during flexi-bar exercise. J Phys Ther Sci, 2015, 27: 1889-1891. [Medline] [CrossRef]

14) Cools AM, Dewitte V, Lanszweert F, et al.: Rehabilitation of scapular muscle balance: which exercises to prescribe? Am J Sports Med, 2007, 35: 1744-1751. [Medline] [CrossRef]

15) Kronberg M, Németh G, Broström LA: Muscle activity and coordination in the normal shoulder. An electromyographic study. Clin Orthop Relat Res, 1990, (257): 76-85. [Medline]

16) Kuechle DK, Newman SR, Itoi E, et al.: The relevance of the moment arm of shoulder muscles with respect to axial ro- 
tation of the glenohumeral joint in four positions. Clin Biomech (Bristol, Avon), 2000, 15: 322-329. [Medline] [CrossRef]

17) Lim JY, Lee JS, Mun BM, et al.: A comparison of trapezius muscle activities of different shoulder abduction angles and rotation conditions during prone horizontal abduction. J Phys Ther Sci, 2015, 27: 97-100. [Medline] [CrossRef]

18) Saha AK: Mechanism of shoulder movements and a plea for the recognition of "zero position" of glenohumeral joint. Indian J Surg, 1950, 12: 153-165. [Medline]

19) Ludewig PM, Cook TM, Nawoczenski DA: Three-dimensional scapular orientation and muscle activity at selected positions of humeral elevation. J Orthop Sports Phys Ther, 1996, 24: 57-65. [Medline] [CrossRef] 